\title{
Verdi e Dante. Alcune nuove riflessioni
}

\author{
Antonio Rostagno \\ Università di Roma "La Sapienza" \\ antonio.rostagno@uniroma1.it
}

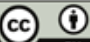

\section{Riassunto}

Viene ripercorso il rapporto di Giuseppe Verdi con alcuni testi a vario titolo collegati a Dante, dalla vicenda della sua prima opera, Oberto conte di S. Bonifacio dove appare Cunizza da Romano, attraverso altre composizioni da concerto, fino alle Laudi alla Vergine Maria sulla preghiera di San Bernardo, da Paradiso XXXIII. Vengono portate alcune nuove documentazioni e proposte alcune nuove interpretazioni.

Parole chiave: Verdi, Dante, Italia, Madrigalismo.

\begin{abstract}
The relationship of Giuseppe Verdi with several texts by Dante is well known, from the history of his first opera, Oberto conte di S. Bonifacio in which Cunizza da Romano appears, to other concert compositions, above all Laudi alla Vergine Maria, San Bernardo's prayer in Paradise XXXIII. This paper contributes with new documents and proposals for a new interpretation.
\end{abstract}

Keywords: Verdi, Dante, Italia, Madrigalism. 
I rapporto di Giuseppe Verdi con la poesia di Dante è assai lungo, tanto da coprire quasi tutta la sua vita compositiva, sia pur con larghissime pause. Forse soltanto verso Manzoni e Goethe possiamo registrare un tanto precoce interessamento da parte di Verdi: di Manzoni infatti ancor prima del debutto operistico pone in musica alcuni cori dalle due tragedie e Il cinque maggio, poi nel 1858 come omaggio all'amico napoletano Melchiorre Delfico scrive una breve lirica sulla terza strofa del coro che descrive la morte di Ermengar-

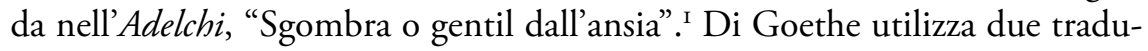
zioni per altrettante liriche da camera pubblicate nel I 838 dall'editore Canti di Milano: Perduta ho la pace e Deh, pietoso, oh addolorata, ${ }^{3}$ testi entrambi provenienti dal personaggio di Gretchen dal Faust, nella traduzione italiana (non eccelsa) di Luigi Balestra. Nonostante la loro semplicità musicale, sono due liriche importanti non solo perché anticipano alcuni incisi melodici poi confluiti nelle opere più mature, ma soprattutto perché affrontano per la prima volta un tema che accompagnerà Verdi molto a lungo: il tema dell' "eterno femmineo", quell'ewig Weibliche per cui a Goethe (dichiaratamente, pur differenziandosene) non era estranea la memoria dei canti conclusivi della Commedia dantesca.

Sin dai primissimi anni, quindi, si chiarisce questa costellazione di rimandi culturali, che testimoniano come Verdi abbia assimilato già durante la formazione i grandi esempi della letteratura europea, condividendo quindi un'educazione culturale solitamente riservata ai letterati più che ai musicisti. Dante costituisce poi per Verdi, come per Donizetti, un modello e un esempio di letteratura nazionale e di altissimo prestigio, a cui essi guarderanno non come a un serbatoio di situazioni, vicende, personaggi o testi da musicare, ma come un esempio di impegno e dignità artistica.

I. La prima apparizione di un personaggio legato alla Commedia nel teatro di Verdi avviene già nella sua prima opera, Oberto conte di S. Bonifacio (I839): il personaggio di Cuniza, l'antagonista femminile dell'opera, proviene infatti da

I. L'interessante relazione di Verdi con Manzoni, per cui nutriva una fanatica ammirazione, è descritta da Ortombina 1992. E a questo studio si rimanda per ulteriori notizie e riferimenti documentari relativi alle composizioni giovanili a cui qui faccio riferimento, composizioni che probabilmente sono ancora conservate dagli eredi a Villa Verdi a Sant'Agata, ma delle quali non si ha altre notizie che alcuni brevi accenni nella corrispondenza verdiana, puntualmente ricostruita da Ortombina.

2. È la traduzione italiana della canzone di Gretchen "Meine Ruh ist hin" dal Faust (Gretchen Stube, vv. 3374-3413), che anche Schubert (Gretchen am Spinnarade, op. 2, Dir8), Wagner e altri hanno posto in musica.

3. È la preghiera davanti alla Mater dolorosa in cui Goethe scrive un liberissimo adattamento tedesco dello Stabat Mater di Jacopone (Faust, Zwinger, vv. 3587-36r19); Schumann ne offre una sublime intonazione nelle Faustscenen aus Goethes Faust, parte prima, seconda sezione. 
Paradiso IX I3-66. È vero che la geometria drammatica e lo sviluppo della vicenda hanno un collegamento solo parziale con la figura che nella Commedia parla a Dante e Beatrice, e che la vicenda portata in scena da Verdi è in larga parte frutto di invenzione: ${ }^{4}$ non si parla della storia del rapimento di Cunizza (nella grafia geminata, il libretto di Verdi opta invece per la lezione scempia); 5 non compaiono né Sordello, altro personaggio dantesco che entrerà nel melodramma italiano dell'Ottocento, ${ }^{6}$ né Ezzelino; non si fa riferimento al suo triplice matrimonio né alla fama di donna spregiudicata, forse proprio per avvicinarsi alla figura celestiale che Dante tratteggia. Cunizza da Romano non è figura marginale nella Commedia; collocata fra gli Spiriti amanti nel terzo cielo di Venere, la sua luce attira Dante:

Cunizza fui chiamata, e qui refulgo perché mi vinse il lume d'esta stella (Pd. IX, 32-33),

cui segue la profezia sulla Marca Trevigiana e su Feltre:

Piangerà Feltro ancora la difalta de l'empio suo pastor, che sarà sconcia sì, che per simil non s'entrò in malta. (Pd. IX, 52-54)

Oltre al citato Sordello, Dante aveva incontrato anche Ezzelino III, lo spietato fratello di Cunizza (che Verdi tuttavia non porta in scena), fra i violenti contro il prossimo, nel primo girone del settimo cerchio dell'Inferno (If. XII IO9-IIO). Il personaggio storico di Cunizza da Romano (II9O-I279) è passato alla storia

4. Si potrebbe perciò pensare che la figura dantesca e l'eroina verdiana non possano essere identificate; ma se così ragionassimo, anche la Pia de' Tolomei e la Francesca da Polenta, figure che hanno avuto molteplici intonazioni da parte dei compositori italiani, non sarebbero ascrivibili al modello di Dante. Il libretto di Cammarano per l'omonima opera di Donizetti infatti non deriva dai pochi versi di $\mathrm{Pg}$. V I30-136; al contrario, il personaggio donizettiano come le altre Pie del melodramma italiano medio-ottocentesco derivano dal romanzo di Bartolomeo Sestini (I822), dal dramma di Giacinto Bianco (I836) o da quello di Carlo Marenco (1838). Similmente il libretto della Francesca da Rimini di Felice Romani (poi utilizzato da diversi compositori) proviene dal dramma di Silvio Pellico più che dal modello poetico di Inferno V. Eppure è comune convinzione, pienamente condivisibile, che la fortuna di queste figure nell'Ottocento debba essere posta in relazione con l'evidentissimo movimento di interesse per Dante caratteristico dell'intera cultura romantica italiana, non solo del melodramma. Data questa situazione, non si ritiene necessaria altra cautela nell'indicare la discendenza, sia pur indiretta, della Cuniza dell'Oberto dalla suggestione dantesca.

5. Non entriamo qui nel problema dell'attribuzione del primo libretto di Verdi, nel quale il ruolo di Antonio Piazza e quello di Temistocle Solera non sono del tutto chiariti; la bibliografia è ampia e si rimanda alla voce curata da Ottomano 20I3, con sintetici riferimenti bibliografici.

6. Sordello. Opera in quattro atti, libretto di Temistocle Solera, musica di Antonio Buzzi, Milano, Teatro alla Scala, Carnevale/Quaresima I856-57 [Milano, Pirola, I856]; come ci si può aspettare, il libretto reca nel frontespizio una breve citazione da Pg. VI 74-75 (“... Io son Sordello / della tua terra"). 
come donna diabolica, assetata di potere, sanguinaria e soprattutto libertina; è probabile che nella parte finale della vita Cunizza avesse subito un pentimento e dal 1265 al 1279 potrebbe aver vissuto a Firenze, dedita a opere pie, dove Dante stesso avrebbe potuto conoscerla. La scelta di Dante di inserirla nel Paradiso ha destato a lungo incertezze di interpretazione. Già nel Discorso (capp. XLVI-XVLII) Ugo Foscolo (I825: 229-23I) aveva giustificato la sua presenza con motivazioni politiche anti-guelfe (Foscolo è spesso incline a credere che le scelte di Dante siano motivate di anno in anno dalle vicende contingenti, e il caso di Cunizza sarebbe una di queste).

Nell'opera di Verdi, Cuniza (mezzo-soprano) è il vero personaggio positivo, protagonistico; è l'unica che, come accadrà ai suoi eroi della maturità, compie una scelta, e questa scelta non è motivata dall'utilitarismo individualistico, ma dal fine di ottenere il bene altrui, secondo i principi del liberalismo etico ottocentesco condiviso da Verdi. Cuniza infatti sceglie di rinunciare al promesso sposo Riccardo (tenore) quando apprende di un suo precedente impegno con Leonora (soprano), figlia del proscritto Oberto (basso), e agisce per realizzare questa scelta di sacrificio del proprio bene personale a favore di una giusta causa. Non desta quindi sorpresa il fatto che Verdi abbia lavorato molto sulla sua parte; nei due anni che seguirono la prima milanese rielaborò molte delle parti dove Cuniza è coinvolta: una nuova aria, un nuovo duetto con il tenore, ma soprattutto un duetto che compare nell'autografo, ma non nella versione definitiva, con la antagonista Leonora, nel quale Verdi traduce il senso della compassione di Cuniza, che porta le due donne non solo a comprendersi, ma persino ad abbracciarsi, riconoscendo la propria comune posizione di vittime della situazione (Budden 1992: 43-67, partic. 65-67).

Non occorre qui riassumere la vicenda, sia però sufficiente ricordare che Verdi ne fa un'eroina positiva, e in lei rappresenta il tema del perdono, già caro a Donizetti (Marin Faliero, Maria Stuarda, Pia de' Tolomei ecc.), di ovvia ascendenza manzoniana (soprattutto il Carmagnola, ma è tema radicato nella morale giansenista). Occorre però ricordare che Verdi è all'inizio della carriera e non ha ancora l'autorità per imporre ai librettisti le sue opinioni drammaturgiche; la scelta del personaggio dantesco quindi è più una casualità che una deliberata intenzione. Ma non per questo tale scelta sembra meno significativa.

Rimane sorprendente come già in questo primo dramma Verdi abbia utilizzato l'ispirazione dantesca (e non è importante il fatto che il giovane Verdi non avesse ancora influenza sulla stesura del libretto; importante è invece che la sua ispirazione musicale si concentri su questa figura per realizzare un personaggio già completo). E ugualmente degno di nota è che la Cuniza del I839 già lasci intravedere il processo di nobilitazione morale del personaggio attraverso sofferenze e sacrifici, come sarà di molti futuri protagonisti verdiani, soprattutto femminili. Questo lascia intendere che lo stesso Verdi, come 
Goethe, vede nella donna dantesca un simbolo di redenzione collettiva da parte dell'elemento femminile; vede in altri termini un tema analogo all'ewig Weibliche goethiano, dove la lettura sotto l'ottica del personaggio dantesco costituisce l'inizio di un lungo percorso.

2. Dal 1842 Verdi imbocca la via dell'impegno politico-civile; in modo esplicito a partire da Ernani, il primo libretto per cui ha una certa libertà di scelta e sul quale può intervenire già nella fase preparatoria, ${ }^{7}$ Verdi predilige argomenti di più decisa avanguardia, volgendo attenzione a figure di ribelli create da Victor Hugo o da Byron, o a personaggi e situazioni di maggiore impegno come Attila o Giovanna d'Arco, o infine a tipi umani estremi come Macbeth. Da questo momento, e così sarà per lunghi anni, l'urgenza del messaggio lascia spazio nel teatro verdiano per più approfondite meditazioni su personaggi danteschi. Nello stesso tempo, indipendentemente da Verdi, si avvia nella romanza italiana più di consumo la voga di utilizzare testi danteschi ma non tratti dalla Commedia. La romanza italiana fra anni Quaranta e fine secolo predilige testi del Dante più prossimi al modello stilnovista, il Dante di alcuni punti della Vita nuova; in particolare si registra una profusione di intonazioni sul sonetto Tanto gentile e tanto onesta pare. Verdi è del tutto immune da questa moda, poiché si tratta davvero di una moda, contagiosa e persistente, che giunge fino al Novecento. ${ }^{8}$ Non è scopo di questo scritto stilare un elenco completo delle intonazioni dantesche, basti indicare come nello stesso Ottocento, a partire da anni precedenti il debutto di Verdi e fino alla fine della sua vita creativa, in termini numerici le composizioni $s u$ testi o ispirate a testi danteschi aumentano continuamente. Ma i musicisti sembrano considerare solo una ristrettissima selezione di estratti: oltre a quelli già indicati, basti qui ricordare l'episodio di Paolo e Francesca, il brevissimo della Pia (che però entra nella musica attraverso la tragedia in versi di Carlo Marenco, non per i pochi versi che Dante le riserva), Ugolino, il Pater noster del canto XI del Purgatorio, più sporadicamente Per una ghirlandetta e poco altro.

7. È da tempo noto il carteggio con il librettista Francesco Maria Piave (la pubblicazione più completa è in Conati 1983), al quale Verdi può imporre scelte di situazione, di versificazione, di taglio delle scene, insomma di drammaturgia, in modo molto più incisivo di quanto potesse fare con Temistocle Solera. E ciò avviene sin dalla scelta del soggetto: è vero che la proposta iniziale giunge dalla Presidenza del teatro La Fenice di Venezia, per cui Verdi scrive l'opera, ma è Verdi stesso che, dopo avere scartato almeno due precedenti proposte, accoglie con entusiasmo l'idea dell'Hernani. Nei quattro precedenti suoi titoli (Oberto, Un giorno di regno, Nabucodonosor, I Lombardi alla prima crociata) Verdi aveva solo preso il libretto finito impostogli dall'impresario milanese Lanari, secondo la prassi di quegli anni.

8. Un parziale catalogo della musica a vario titolo legata a Dante si trova in Roglieri 2000; più recente è poi Roglieri 20I2. Qualche commento ad alcune liriche italiane su testi danteschi è poi in Frantellizzi $201 \mathrm{I}$. 
Perché Verdi torni a Dante occorre attendere assai a lungo, e non sarà nel campo del melodramma, ma in quello della musica da concerto. Saltiamo infatti dal I839 al I880, quarant'anni che hanno profondamente modificato la lettura di Dante da parte dei commentatori italiani, ma soprattutto hanno modificato l'assetto sociale e culturale nel contesto nazionale. L'immagine di Dante non è più quella di Foscolo e Mazzini, monumento di idealità, la cui levatura artistica non è riconosciuta primariamente nella perfezione del verso, della forma poetica, quanto nella profondità del pensiero tanto politico quanto filosofico. Nella Nuova Italia a partire dal 1860 molto è cambiato, e Verdi come Dante sono ora due personaggi simbolici, nella figura, fama e interpretazione dei quali sono intervenute altre motivazioni e altri contenuti collettivamente condivisi.

$\grave{E}$ in questo mutato clima che Verdi decide di porre in musica due testi sacri, due preghiere che egli crede "volgarizzate" da Dante, ossia da lui tradotte dal latino. Si tratta della Ave Maria per soprano e archi e del Pater noster per coro a cinque voci a cappella (prima esecuzione: Milano, La Scala, I88o). Oggi sappiamo bene che nessuno dei due testi è dantesco, e avrebbe dovuto saperlo anche Verdi dato che entrambe le attribuzioni erano state da tempo chiarite. Ma per ognuno di essi è facile ricostruire i motivi di questa errata attribuzione a lungo vigente, e forse anche individuare le fonti da cui Verdi attinse il testo musicato, trasmettendo al grosso pubblico ancora per decenni la vecchia attribuzione nonostante il chiarimento dei filologi. Concentreremo qui l'attenzione sulla Ave Maria, poiché lo stesso testo sempre con attribuzione dantesca era già stato utilizzato da Donizetti e tornerà a fine secolo ad essere impiegato anche da Riccardo Zandonai. ${ }^{9}$

Donizetti aveva incontrato questa "volgarizzazione" di Dante al termine della sua carriera compositiva, nel I844, quando era Maestro della Camera di S. M. l'Imperatore d'Asburgo (Hofkapellmeister) a Vienna. L'incarico prevedeva che il compositore scrivesse anche musica per funzioni religiose, ed è in questa produzione che troviamo una Ave Maria, offertorio per Soprano, Contralto e Archi. La breve composizione fu eseguita a Vienna nei "Concerti Spirituali della Settimana Santa" dello stesso I844; non ho potuto accertare altre esecuzioni fino al I866, quando venne replicata a Roma in un concerto assai particolare, dove Giovanni Sgambati diresse la prima esecuzione completa della versione definitiva della Dante-Symphonie di Franz Liszt, presente all'esecuzione (cfr. De Angelis 1935). Il testo è chiaramente il medesimo che

9. Zandonai, prima della celebre opera Francesca da Rimini del I9I4, che tuttavia non si ispira ai personaggi danteschi, ma proviene dalla penna di Gabriele d'Annunzio, librettista insieme a Tito Ricordi, sin dagli anni giovanili è lettore assiduo della Commedia. Su testi danteschi oltre all'Ave Maria, sono infatti il Pater noster (Pg. XI) a quattro voci maschili e orchestra (I899, pubbl. 1913), le Scene dal V Canto dell'Inferno ("O anime affannate"; I899, pubbl. 1964) e la cantata Il conte Ugolino, composta nel gennaio 1900 (Cescotti 1999). 
trentasei anni dopo utilizzerà anche Verdi, sia pur con lievi varianti; occorre una piccola digressione su questo presunto "volgarizzamento" dantesco.

Questa Ave Maria è la quinta preghiera mariana composta da Donizetti, la prima risaliva al lontano I8I9; ma solo per quest' ultima del I844 la stampa reca la indicazione "Tradotta da Dante" (l'edizione in riduzione pianistica fu fatta molto più tardi, nel I865 dall'editore Lucca di Milano, n.e. I5345). Una lettera di Donizetti all'amico-allievo bergamasco Adelson Piacezzi chiarisce la fonte d'origine del testo, un'edizione della Commedia con il commento di Cristoforo Landino del $1497,{ }^{\text {IO }}$ contenente nelle appendici un Credo "volgarizzato da Dante"; ${ }^{\text {II }}$ sia il Pater noster sia, nella parte conclusiva, l'Ave Maria sono estratti da questo Credo, e Donizetti subito pensa a porli in musica:

Di qual gemma mi hai fatto possessore! Ho amato (se non sempre compreso) Dante, ma tu col tuo dono raddoppiasti l'affetto e la venerazione. Ti accuserei quasi di crudeltà se in altre mani fosse caduto. L'amicizia che ci legò fin dai primi anni di nostra giovinezza ti parli in mio favore (lett. $n$. 532 ad Adelson Piacezzi, 9 gennaio I844; Zavadini 1948: 124).

Nel I88o Verdi riprende il medesimo offertorio mariano in una composizione ingiustamente trascurata dagli storici. L'intero capitolo ternario da cui tale testo è tratto (l'inizio recita: "Io scrissi già d'amor più volte rime / quanto più seppi dolci, belle e vaghe") fu a lungo considerato una traduzione dal latino dovuta a Dante, forse anche per l'uso della terza rima non frequente nelle preghiere, seguendo l'attribuzione delle edizioni quattrocentesche sopra citate. Ma nel I844, quando Donizetti lo intona, era da tempo chiarita l'attribuzione al maestro Antonio da Ferrara. Sin dalla sua Drammaturgia (I666) Leone Allacci aveva eliminato ogni dubbio; eppure ancora nel medio Ottocento diversi testi italiani conservarono l'attribuzione a Dante. L'edizione Zatta delle Prose e rime liriche edite ed inedite di Dante Alighieri con copiose ed erudite aggiunte (tomo IV, parte II, Venezia, I758) dedicava un ampio commento al Credo non accennando neppure di sfuggita a una possibile attribuzione alternativa: Il Credo di Dante Alighieri, illustrato con annotazioni dell'Abate Francesco Saverio Quadrio (pp. 235-262). La stessa attribuzione dantesca del Credo ricorre anche nel Saggio di rime di diversi buoni autori, che fiorirono dal XIV fino al XVIII secolo (Firenze, Ronchi e C., I825), curato da Luigi Rigoli. E ancora nel I839, vicino quindi alla composizione di Donizetti, l'accademico della Crusca Bartolommeo Gamba, che cita Rigoli, ne ripete l'attribuzione a

Io. Comento di Cristoforo Landino Fiorentino sopra la Commedia di Dante Alighieri, Venezia, Giunta, I497; che riprende l'edizione del I49I con aggiunta de: "il Credo, il Paternostro, e l'Avemaria di Dante". La nota dichiara la discendenza di queste aggiunte dall'edizione che cito alla successiva nota.

II. La prima edizione della Commedia che attribuisce questa "volgarizzazione" a Dante è quella di Bernardino Benalj e Matteo da Parma (Venezia, I49I), che include nelle ultime pagine "il Credo, il Paternostro, e l'Avemaria di Dante" con postille. 
Dante. ${ }^{\mathrm{I2}}$ Ma poco dopo, nel Saggio di Rime di Dante, di Maestro Antonio da Ferrara, di Franco Sacchetti, di Luigi Pulci, del Poliziano, di Feo Belcari [...] curato da Anton Maria Salvini (Firenze, Piazzini, I847, pp. 23-24), leggiamo:

Maestro Antonio da Ferrara oltre la Canzone fatta a Dio, essendo nello stremo di sua vita dolendosi de' suoi peccati, espresse l'Ave Maria e 'l Pater noster, la Salve Regina. Ed è da notare che il Credo ascritto in alcuni Manoscritti e libri stampati a Dante è di questo Antonio, secondoché si vede specificamente notato nel volume delle Laudi de' Bianchi, delli quali si darà notizia più particolare a suo luogo. D'onde di più s'ha che l'Ave Maria recitata a quei tempi, è simile a quella che oggidì recita la Chiesa, contro l'opinione d'alcuni, che asseriscono la metà di detta orazioncella non essere più anziana di Leone $\mathrm{X}$.

Con questa riattribuzione corretta si potrebbe pensare che la controversa vicenda sia giunta al termine; ma ancora nel 1865 un anonimo commentatore attivo a Firenze stampa La professione di fede di Dante Alighieri o parafrasi in terza rima del Credo, de' Sacramenti, del Decalogo, dei Vizi capitali, del Pater Noster e dell'Ave Maria (Firenze, a spese della Società Toscana per la diffusione di buoni libri, Tipografia delle Murate, 1865): la "professione" contenente il testo mariano è indicata sin nel titolo ("di Dante Alighieri"). Con ogni probabilità il testo della composizione di Verdi del i88o proviene da questa edizione. La seguente tabella visualizza le differenza fra le varie "volgarizzazioni":

\begin{tabular}{|c|c|c|c|c|}
\hline Saggho di rime (1825) & Donizetti (1844) & Professione di fede di D.A. (1865) & Verdi (1880) & \\
\hline 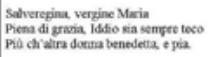 & 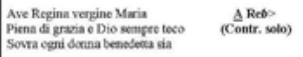 & 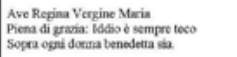 & 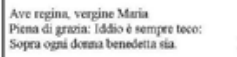 & $\begin{array}{l}\Delta s k \\
D o s(V)\end{array}$ \\
\hline 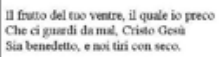 & 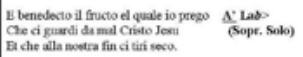 & 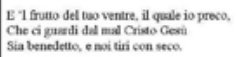 & 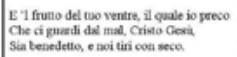 & $\begin{array}{l}\text { prat< } \\
\text { Fats }\end{array}$ \\
\hline 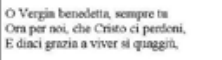 & 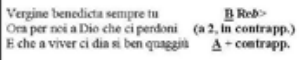 & 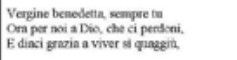 & 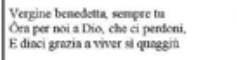 & $\subseteq \$$ \\
\hline Che Parativo al notro fin di doni. & $\begin{array}{l}\text { Che alla fin Parasiso di deni } \\
\text { [Ave Regina, piena di graia] }\end{array}$ & Che 1 Paradiso al nostro fin di doni. & $\begin{array}{l}\text { Che IParafiso a nostro fin d doci } \\
\text { [Ave Regina Ora per nat a Dio] }\end{array}$ & $\begin{array}{l}\text { p } \mathbf{S}< \\
|A| s \mid>\end{array}$ \\
\hline
\end{tabular}

Tabella comparativa delle edizioni dell'offertorio in forma di capitolo ternario.

Legenda (per le parti musicali): Contr. $=$ contralto; Sopr. $=$ soprano; contrapp. $=$ contrappunto; le lettere maiuscole sottolineate indicano i temi musicali, ricorrenti in Donzietti, sempre rinnovati in Verdi (forma strofica vs. forma durchkomponiert).

Potremmo procedere nella vicenda di questa strana attribuzione che non vuole staccarsi da Dante; ma sia qui sufficiente quanto detto per capire come sia

I2. Gamba da Bassano I839: 335b (n d'inventario ro9r: "Il Credo ed un Sonetto" ); qui, nella sezione di attribuzioni dantesche si legge: "Esistono due edizioni antiche [del Credo], una del secolo XV, in forma di $4^{\circ}$, in carattere tondo; ed una del secolo XVI, in $4^{\circ}$. In questa seconda, dopo le parole CREDO DI DANTE, vedesi un intaglio in legno che rappresenta Dante in atto di salire il monte, colle tre fiere che gli si fanno incontro, come le descrive al canto I dell'Inferno. L'una e l'altra edizione stanno alla Trivulziana. Sì il Credo che il Sonetto si pubblicarono con illustrazioni anche nel Saggio di Rime di diversi buoni Autori ec.; Firenze, Ronchi e Compagni, I825" (la fonte dichiarata per l'attribuzione è "G-A.", ossia lo stesso Gamba da Bassano I828). 
stato possibile che ancora Donizetti e Verdi abbiano in buona fede potuto credere di usare parole di Dante ancora nel i844 e nel I88o. Quando l'editore Ricordi stampa i due pezzi di Verdi (Ave Maria e Pater noster), il frontespizio reca senza ambiguità "volgarizzati da Dante"; non dubitiamo della buona fede di Verdi, ma occorre ricordare che con queste composizioni egli stava attuando un disegno politico, o meglio di politica culturale. Nel I88o siamo solo due anni prima della Triplice Alleanza con la Prussia e molti vecchi intellettuali, fra cui Verdi in prima linea vedono un rischio di indebolimento della formazione del comune senso della nazione, realmente ancora molto labile nella Nuova Italia. Per questo Verdi non manca di ripetere, insieme al timore per progressiva "germanizzazione", la necessità di affermare anche presso il pubblico più vasto, verso ogni strato sociale, verso l'intero corpo della nazione, un patrimonio comune di cultura nazionale con alcuni suoi pilastri centrali: Dante anzitutto, poi Petrarca, Palestrina per la musica, Manzoni per la narrativa, se stesso per il melodramma. La scelta delle due preghiere "volgarizzate da Dante" risponde a questo impegno: se i temi della Commedia non sono considerati utilizzabili dal drammaturgo, il Poeta può però offrire materia per composizioni da concerto. E con questa intenzione nascono le due preghiere.

E altresì poco probabile che Verdi conoscesse l'offertorio mariano del tardo Donizetti. Un confronto fra le composizioni dei due musicisti indica, al di là delle intenzioni culturali di Verdi, due sensibilità religiose e due concezioni della spiritualità del tutto opposte, mostrando già come Verdi stesse confrontandosi con i temi della fede e del culto mariano con un impegno e una profondità critica impensabile per Donizetti. Comparando i due inizi, la differenza è chiara:
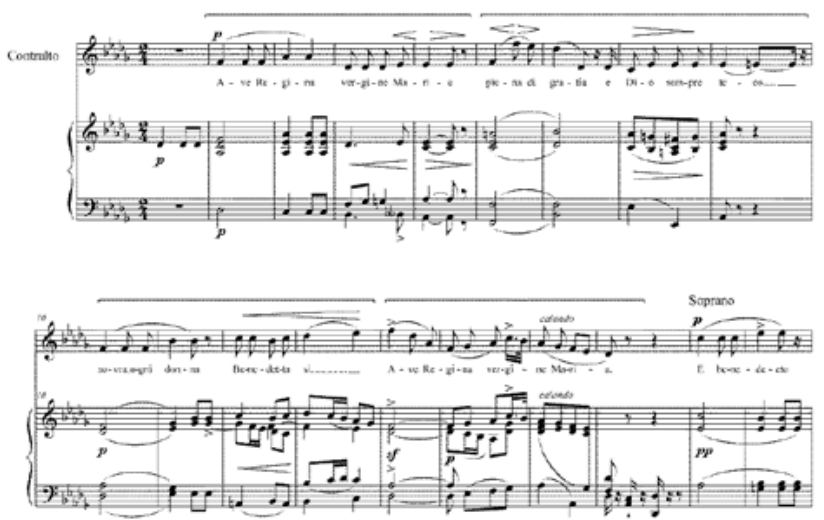

Es. Ia, G. Donizetti, Ave Maria (1844), inizio (bb. I-8) 

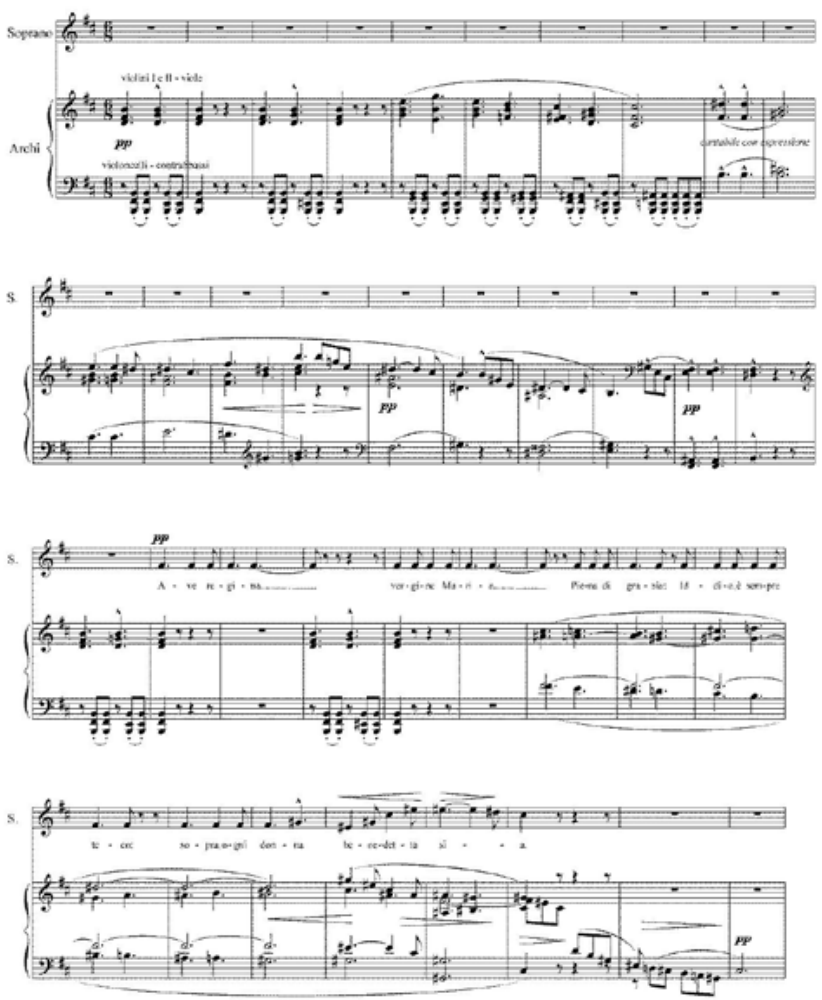

Es. Ib, G. Verdi, Ave Maria (188o), inizio (bb. I-37)

Quella di Donizetti è una semplice melodia, progettata secondo le consuete simmetrie periodali, elegante se si vuole ma evidentemente priva di intenzione significante; in secondo luogo è in forma strofica, ossia le musica si ripete uguale per le diverse strofe: la prima è esposta dal contralto alla tonica principale (Re bemolle maggiore), la seconda dal soprano (in La bemolle maggiore; ultime due battute dell'es. Ia); la terza ancora dal contralto, con un contrappunto del soprano. In tal modo viene semplicemente sonorizzato il percorso dal "viver ben quaggiù" al "paradiso" declamato in conclusione. È insomma una semplice e fideistica religiosità, una fede un poco convenzionale, che non esprime alcuna posizione critica, alcuna riflessione individuale del compositore. Opposta è la spiritualità tormentata da dubbi e timori di Verdi, che si ripercuote profondamente nella sintassi musicale, piegandola a esprimere l'intenzione critica-dottrinaria del compositore. I minacciosi accordi dissonanti con cui si apre la sua preghiera sono inequivocabili; ancor prima che la voce inizi l'invocazione, l'oscurità e il turbamento non lasciano filtrare alcuna luce della fede. 
La composizione viene scandita da Verdi in quattro fasi: nella prima la voce è quasi fissa sul Fa diesis e avvolta dal timbro scuro e grave degli archi, continuamente attraversato da dissonanze e cromatismi: improvviso giunge uno squarcio di luce alle parole "benedetta sia" con un inarcamento della voce verso l'acuto, subito riportato nel registro centrale. Un assai simile processo di significazione del testo musicale si ritrova nel Pater noster, quando alla quarta misura le voci acute si distaccano dall'unisono/ottava, in scansione omoritmica e in una disposizione stretta nel registro centrale (Re2-Re3), per elevarsi verso i registri superiori, raffigurando il volgersi della comunità orante ai "cieli" da cui il padre guarda l'umanità:

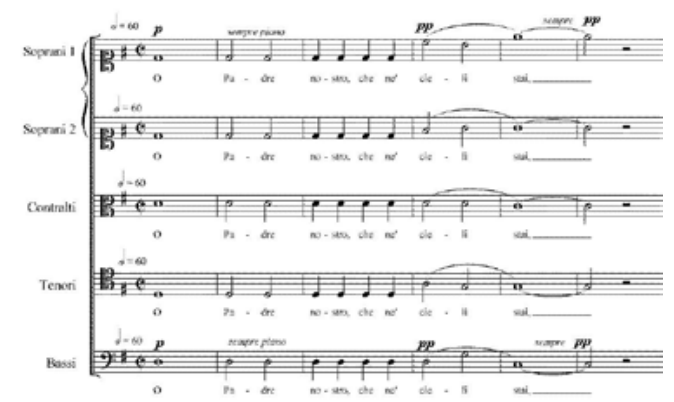

Es. 2, G. Verdi, Pater noster (I88o), inizio (bb. I-6)

La seconda strofa dell'Ave Maria verdiana, in Fa diesis minore, colloca la voce in un registro medio-acuto più chiaro, raffigurante l'apertura a una possibile speranza in Cristo. La terza strofa è quella più melodica, cantabile e in tonalità maggiore $(\mathrm{Si})$, dove l'armonia meno cromatica acuisce il senso di pacificazione: è la strofa in cui l'orante chiede "Vergine benedetta, sempre tu, / ôra per noi a Dio che ci perdoni / E diaci grazia a viver sì quaggiù / che 'l Paradiso al nostro fin ci doni", il solo momento in cui viene espressa la speranza nella redenzione dell'umanità grazie all'eterno femmineo. Ma l'offertorio non viene terminato qui da Verdi, che assai significativamente ripete la strofa iniziale riportando così nella musica lo stesso stato di oscura incertezza dell'inizio: di nuovo, dopo l'espressione della speranza, l'uomo è ricondotto in uno stato in cui non vede la luce della fede, non accede alla via luminosa del "Paradiso" promesso. Ciò avviene quando le parole "ôra per noi" vengono ripetute, nella conclusione, più e più volte, ma senza alcuna convinzione nella speranza di una grazia, interrotte e quasi "risucchiate" da pause cariche di dubbi e dalle sonorità oscure dell'inizio. È lo stesso dubbio, la stessa paura del singolo uomo, che dalla vita mondana guarda con timore l'eterno senza ottenere alcuna luce di speranza, che aveva contraddistinto le parti più tragiche del Requiem, solo sei anni prima, come il Dies irae, Mors stupebit o soprattutto il Libera me fi- 
nale. ${ }^{13} \mathrm{Nel}$ volgersi alla Vergine questo povero uomo del dubbio affida le sue poche speranze, come Goethe, all'eterno femmineo; ma mai troverà la luce della certezza, che invece Faust troverà nelle ultime scene del dramma, rivedendo Margherita trasfigurata ("una delle penitenti, una volta chiamata Margherita") e il coro mistico. La voce femminile che declama l'Ave Maria di Verdi prefigura dunque quel soprano solo che declamerà l'invocazione conclusiva del Te Deum, l'ultima composizione di Verdi, "In te Domine speravi": è la voce dell'uomo solo che non ha altra certezza se non la "paura" di cui parla lo stesso Verdi secondo una testimonianza diretta. ${ }^{\mathrm{I}} \mathrm{E}$ E chiaro, a questo punto, che Verdi non condivide, o meglio non sente il messaggio finale della Commedia, nulla lo porta alla luce smaterializzata della fede; ma da laico, da uomo che ha attraversato le lotte del Risorgimento, il liberalismo umanitario, la fiducia nella formazione di un uomo nuovo in un mondo nuovo, l'estasi mistica non è cosa per lui, non la può sentire. E infatti nulla della fede che stordisce per troppa luce passa nella sua musica, appesantita al contrario da voragini di oscurità, di dubbio esistenziale, di sospensione della fede da parte di un uomo che pur l'ha cercata a lungo. Insomma: il percorso spirituale che Dante compie nella Commedia e il percorso esistenziale di Verdi vanno in direzioni opposte.

Nel quarto atto di Otello (opera d'altronde ricca di risonanze dantesche nel libretto di Arrigo Boito), ${ }^{\text {Is }}$ di sette anni successivo all'Ave Maria per soprano e archi, Verdi compone una nuova Ave Maria, recitata da Desdemona prima di coricarsi, pochi minuti prima di essere uccisa. In Shakespeare questa scena non c'è, né Rossini nel suo omonimo melodramma aveva fatto nulla di simile. Le analogie con l'offertorio Ave Maria del I880 sono numerose e significative; le vere e proprie similitudini di materiale musicale non sono molte, eppure il linguaggio musicale è evidentemente affine. Desdemona prega da convinta cristiana, da persona che ha una fede semplice e certa, come Donizetti fiduciosa che la preghiera porti a quel paradiso sperato richiamato nell'ultimo verso. Ma la musica di Verdi comunica esattamente l'opposto: il dubbio, l'assenza di certezze, la paura della giovane donna davanti alla morte. Con la scena che segue, infatti, Verdi mostra che la preghiera e la fede non giungono ad alcun esito: Desdemona (e lo stesso Otello) muoiono per il nulla, per nessun ideale e per nessuna fede, senza alcuna redenzione. "La morte è il nulla", proprio come aveva diabolicamente anticipato Jago. È il pessimismo cosmico di Verdi.

13. Per questa celebre lettura del Requiem cfr. Mila 1980 (in particolare "Verdi sacro", pp. 256-284).

I4. Lo racconta Giuseppe Depanis: "[per questa frase finale del soprano solo] Verdi raccomandava di collocare l'artista il più lontano possibile, nascosta al pubblico, quasi una voce dell'aldilà, voce di sgomento e di supplicazione. 'È l'ümanità che ha paüra dell'inferno', finì per dire a meglio spiegare il concetto appoggiando sulla ü di umanità e di paura [...]”, in Conati 2000: 345 .

I5. Ne parla, fornendone una sommaria ma utile rassegna, D'Angelo 20Io. Recentemente sono tornato su alcuni di questi passi in Rostagno 2010-20II: 36 et passim. 
3. Con tutt'altra profondità, fuori delle luci della ribalta del melodramma e lontano dalla occasione concertistica dell'offertorio del ı88o, Verdi torna a Dante nelle sue estreme composizioni; più precisamente nel terzo dei Quattro Pezzi sacri (pubblicati nel 1898), le Laudi alla Vergine Maria, che intonano le prime sette terzine dell'ultimo canto del Paradiso, la preghiera di San Bernardo. Ed è su questa composizione che occorre ora fermarsi, lavoro di grande impegno intellettuale, scritto più per sé che per l'ascolto pubblico. Un Verdi che nessuno si attenderebbe e che ha davvero lasciato alle spalle la concezione di musica come comunicazione sociale, del melodramma come ammaestramento di vita. È un intellettuale nella sua tarda fase, e come tutti i tardi stili dei grandi artisti imprime una svolta che improvvisamente cancella tutto quanto già fatto e apre prospettive che solo le generazioni a venire potranno recepire.

Le Laudi sono rimaste infatti per più di un secolo un brano assai poco conosciuto, pochissimo commentato e non perfettamente compreso, non eseguito se non in rare occasioni e sempre con mezzi esecutivi non adeguati alle originarie intenzioni dell'autore. Verdi voleva infatti quattro sole "voci bianche", come compare nel frontespizio della prima stampa, ossia voci femminili (non necessariamente fanciulli cantori) ma alleggerite e senza il pesante volume dell'emissione lirica comune al dramma di estrazione verista (ossia, anzitutto, senza quel vibrato persistente e ampio che connota la voce potente del cantante tardo-ottocentesco e novecentesco). Ciò che aveva in mente Verdi qui era piuttosto un neo-madrigalismo, una esecuzione con voci chiare, prive del consueto vibrato e del volume smisurato richiesto dal palcoscenico. Oggi invece le Laudi, in quelle infrequenti occasioni esecutive, si ascoltano eseguite dal coro teatrale composto di 8o-Ioo elementi, dove la meditazione solitaria e intima, quasi una parola interiore, diviene un consueto coro operistico, che a chi non conosca bene il testo poetico potrebbe ricordare qualcosa a mezza via fra il "Va pensiero" e l'"Inno al sole" di Mascagni.

A chi invece ha la fortuna di ascoltare un'esecuzione secondo le intenzioni originali del compositore, ecco che il testo dantesco diviene chiaramente comprensibile e al commento musicale di Verdi viene restituita la qualità di profonda meditazione necessariamente intima, lontano dall'esibizionismo da palcoscenico, espressa in profonda solitudine.

Se mai una composizione di Verdi ha richiesto il commento, l'esame dei particolari, questo è il caso delle Laudi. D'altronde si tratta della sola musica di Verdi che utilizza versi di un grande poeta nati per essere letti e non per essere intonati dal canto, se si escludono quelle prove giovanili ricordate in inizio. Nella sua vita creativa Verdi ha sempre concepito il testo poetico come una base, una impalcatura per la drammaturgia, per il vero testo drammaturgico, che si sarebbe poi affidato esclusivamente alla musica e alla messinscena. Nonostante la qualità dei testi di Boito, anzi direi quasi grazie ad essi, grazie al 
fatto che con Boito per la prima volta Verdi ha potuto disporre di testi di alta ambizione letteraria, è solo con le Laudi che il compositore affronta la grande poesia. Questa è d'altronde una caratteristica della musica vocale italiana ottocentesca, d'opera e no; a differenza della liederistica tedesca romantica, che necessita quasi sempre di testi di grandi poeti, la lirica teatrale o cameristica italiana concepisce il testo come strumento provvisorio, come oggetto non "di" arte ma "su cui" produrre arte: un "pre-testo". Ma il caso delle Laudi è più complesso.

\section{Le Laudi alla Vergine Maria: dal Dante simbolo NAZIONALISTA AL DANTE UNIVERSALE}

Non occorre ripetere che torniamo qui al tema dell'eterno femmineo: ancora una voce umana che si rivolge alla Vergine per ricevere l'illuminazione della grazia, quella grazia che, abbiamo visto, tanto nell'offertorio del i88o quanto nell'atto finale di Otello era scetticamente invocata, ma finiva nel pessimismo che tutto nega, come lo spirito di Mefistofele ("Son lo spirito che nega / sempre, tutto: l'astro, il fior", Arrigo Boito, Mefistofele, Atto I, seconda parte). Entriamo dunque nel testo di Dante e Verdi, un supremo capolavoro che apre la stagione italiana del neo-madrigalismo, destinata ad avere grandi sviluppi fino a Gian Francesco Malipiero e Goffredo Petrassi in pieno Novecento.

Poco sappiamo delle Laudi di Verdi: neppure sulla data di composizione può esserci definitiva certezza, probabilmente collocabile fra il I888 e il I890; il lavoro arriva al pubblico però solo diversi anni dopo, quando su ripetute insistenze di Giulio Ricordi Verdi accetta di inserirlo come numero 3 dei Quattro pezzi sacri. Il compositore aveva pensato queste estreme composizioni come confessioni interiori, riflessioni private, espressioni della sua tarda ricerca di spiritualità e di fede, che non avrebbero dovuto giungere al pubblico, ma finire nella tomba con sé. Le Laudi costituiscono la composizione meno teatrale del ciclo, la più intima; si tratta di un vero e proprio "madrigale spirituale" in un linguaggio musicale rinnovato e attualizzato: ogni immagine del testo, ogni concetto, ogni risvolto etico, filosofico, dottrinario, viene meditato e riespresso con mezzi puramente musicali, senza alcun riferimento all'espressività melodrammatica, presente invece nei due Pezzi Sacri che precedono e seguono le Laudi nel ciclo, lo Stabat Mater e il Te Deum.

Non è forse questa la sede per una analisi minuta; ma sono sufficienti pochi esempi per comprendere la profondità delle intenzioni di Verdi e la raffinatezza di realizzazione. Certo occorre che l'ascoltatore dimentichi il Verdi teatrale e immagini un compositore che non scrive più per i suoi contemporanei, ma medita da solo nel suo studio come Faust, per arrivare a una chiarificazione (prima di tutto a se stesso) della propria spiritualità, del bisogno 
di fede, della necessità di affidare le proprie speranze alla guida della Donna Eterna. Seguirà, nel ciclo, il Te Deum del quale ho già indicato la conclusione negativa, scettica, da parte di un misero uomo che non può avere certezze di fede e percepisce solo il vuoto cosmico. Le Laudi diventano in questo percorso la tappa precedente di questo approdo spirituale negativo; è quindi una tappa non conclusiva, solo una momentanea illusione di poter confidare nelle virtù luminose, benigne, gratuite ("liberali" nel senso etimologico) e amorevoli dell'"eterno femmineo". Ma subito dopo, nel Te Deum appunto, tutto questo si rivela solo illusione: ciò che Verdi compie attraverso i tre ultimi Pezzi sacri, quindi, è il percorso spirituale di un laico che manifesta il proprio bisogno di fede, a lungo cercata ma mai trovata.

Chiudo allora con l'esame di alcuni punti della partitura delle Laudi. Anzitutto Verdi costruisce la sintassi di gran parte della composizione utilizzando con sorprendente ricorrenza il numero tre, il numero simbolico di Beatrice sin dalla Vita Nuova, che su tal numero poggia l'intera sua struttura. Occorrenze ternarie sono disseminate in tutti i parametri nel linguaggio polifonico, ${ }^{16}$ dalla disposizione delle voci all'armonia, dalla ripetizione di parole alla relazione fra le altezze, fino ai canoni che attraversano la composizione (esempi za e 3b).

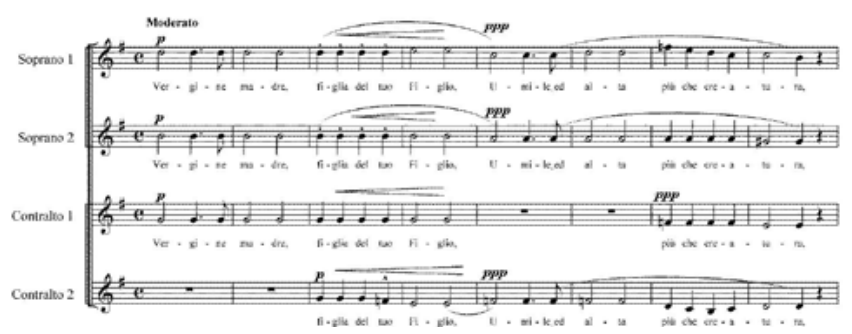

Es. 3a, G. Verdi, Laudi alla Vergine Maria; simbologie ternarie (inizio a tre voci, bb. I-8)

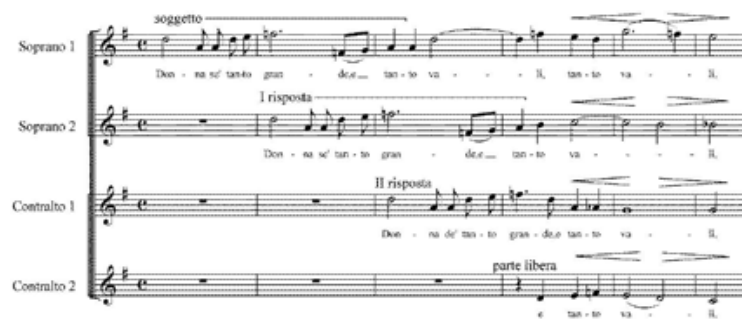

Es. 3 b, G. Verdi, Laudi alla Vergine Maria; simbologie ternarie (canone a tre parti “Donna se' tanto grande", bb. 48-53)

I6. Sulla formazione e i significati dell'armonia triadica nel pensiero cinquecentesco cfr. La Via 2007. 
Nel primo esempio (3a) l'armonia e la declamazione dell'invocazione sono costituite da tre voci a cui la quarta (il contralto secondo) fa da complemento armonico. Nel secondo esempio ( $3 \mathrm{~b}$ ) le entrate a canone coinvolgono le stesse tre voci, lasciando alla più grave la funzione autonoma di sostegno armonico, separandola dall'intreccio della polifonia canonica. Dal punto di vista tecnico, sia sufficiente rilevare come sin dalla battuta 3 il Fa naturale del contralto secondo e la successiva risoluzione a Mi minore spezzi subito i ponti con la tonalità tradizionale e sposti il discorso sul campo modale, che rimarrà fino al termine.

Ogni elemento della sintassi polifonica ha poi il suo significato; il "termine fisso d'eterno consiglio" è identificato dalla "fissità" delle note Mi (primo soprano) e Do diesis (secondo contralto), mentre nelle due voci intermedie l'immagine del disordine della vita terrena provoca un continuo movimento cromatico. Ancor più efficace è l'immagine successiva del "Fattore" (il creatore): tre voci in unisono che in un forte improvviso si aprono ad un sonoro accordo pieno di Mi bemolle maggiore:

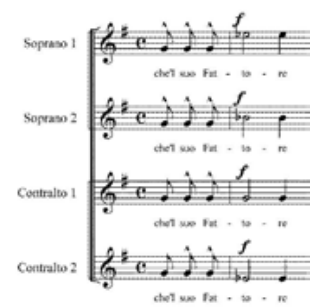

Es. 4, G. Verdi, Laudi alla Vergine Maria; bb. 20-2I

Alla fine della seconda terzina, sull'ultima sillaba accentata Verdi scrive un piccolo melisma, una terzina cantabile alle due voci di soprano ("fattu - - ra"). Questo modo di intonare con melismi più o meno ampi la sillaba che conclude il verso finale di una sezione in terza rima ha origini antichissime: lo troviamo già nei madrigali dell'Ars Nova trecentesca, e lo stesso Verdi lo aveva praticato nelle terzine declamate dai Sacerdoti nella scena del giudizio di Aida (IV atto, scena seconda), dichiaratamente emulanti una terzina dantesca.

"Così è germinato questo fiore": l'immagine ha una lunga tradizione nella letteratura devozionale (non ultimo l'esempio di Jacopone), ma anche nella storia della musica si conoscono mottetti che la tematizzano fin dall'età di Dante stesso (il mottetto politestuale Flos Filium che compare nei Codici di Montpellier per esempio). L'apertura della corolla è sonorizzata dall'allargamento delle quattro voci, che in questo punto giungono a coprire lo spazio di due ottave, molto ampio se consideriamo che Verdi sta scrivendo per voci di tessitura e ambito simile, senza i registri delle voci maschili: 


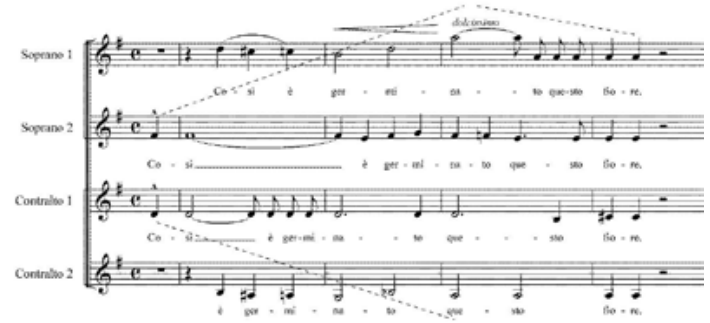

Es. 5, G. Verdi, Laudi alla Vergine Maria; bb. 33-36

L'immagine musicale del fiore che si apre non è rara nella storia musicale antica o contemporanea, anche in compositori assai lontani da Verdi. Per esempio, il verso di Friedrich Hölderlin "Blumen die Menschen, nur blumen..." ("Fiori sono gli uomini, solo fiori..."), ricorre più volte nelle composizioni del compositore ungherese György Kurtág. Lo incontriamo, solo per indicarne una occorrenza, nell'exergo di un frammento del volume 8 degli Játékok (Giochi) intitolato Tanùlmany a Hölderlin-hez... (Studio su Hölderlin: An...). Qui Kurtág scrive una serie di suoni che vanno ampliandosi poco alla volta, in un modo che può curiosamente richiamare l'intuizione di Verdi.

Le successive immagini delle virtù teologali, la "meridiana face di caritate" e la "fontana di speranza", sono sonorizzate da Verdi in modo da richiedere l'esecuzione a voci sole, e che vengono irrimediabilmente compromesse dall'esecuzione di un grande coro. Per l'immagine della "meridiana face" Verdi fa leva su due dati percettivi forti, estremi si direbbe se la parola non fosse contraria allo spirito della composizione: per la prima e unica volta le quattro voci cantano tutta la frase in unisoni e ottave (senza armonia, con forza); il compositore rinnova così un'antica strategia retorica, già utilizzata per esempio da Guillaume Dufay per sottolineare il nome del pontefice nel mottetto Nuper rosarum flores, e poi rimasta in uso fin nell'Ottocento. In secondo luogo la linea melodica disegna un'ascesa in crescendo (registro e intensità quindi collaborano alla realizzazione della luce crescente), fino a raggiungere un La acuto, la stessa altezza che indicava l'apertura del fiore nella precedente immagine del figlio. Ma ancor più interessante è quanto accade dopo il crescendo, con l'immagine della "face di caritate": alla improvvisa ricaduta in registro medio-grave corrisponde l'altrettanto immediata diminuzione di intensità (subito piano) e la chiarificazione armonica nella remota tonalità di Fa diesis maggiore. Non interessa tanto il dato tecnico, di raffinatissima semplicità, quanto l'effetto sonoro che immediatamente rende chiaro il concetto dantesco: la potenza (prima parte della frase) della dolcezza caritatevole (la seconda parte in piano), ossia la forza dell'eterno femmineo. Così Verdi interpreta e realizza in mezzi esperibili dalla pura sensorialità aurale la ossimorica umiltà/altezza, la forza incrollabile e la intimità più riposta. 
L'immagine della "di speranza fontana vivace" è ancor più articolata: dapprima i due contralti in registro grave espongono in imitazione un primo tema sull'intervallo di quinta discendente ("e giuso, in tra i mortali": Do\# - Fa\#, immagine del "basso corporeo", del "quaggiù", della vita mondana); quindi i due soprani entrano con un diverso disegno ascendente sullo stesso intervallo di quinta rovesciato ("se' di speranza fontana": Fa\# - Do\#). Ancora l'ossimorico incontro del "giuso", del mondano, con l'elevazione redentrice dell'"eterno femmineo" (es. 6, secondo sistema), qui nell'immagine della fontana che eleva verso l'alto le speranze della fede.

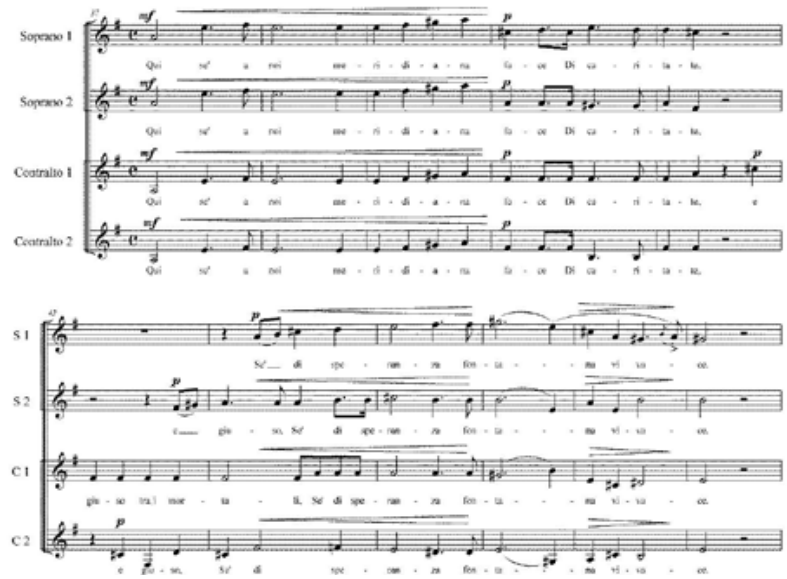

Es. 6, G. Verdi, Laudi alla Vergine Maria ("meridiana face di caritate", bb. 37-4I; "fontana di speranza”, bb. 42-47)

Nella sezione che segue Verdi chiosa, commenta musicalmente il concetto della liberalità, della gratuità: "La tua benignità non pur soccorre / a chi dimanda, ma molte fiate / LIBERAMENTE al dimandar precorre". L'avverbio che ho lasciato in maiuscoletto è da Verdi smisuratamente amplificato per mezzo di una declamazione lenta e molto scandita. Il significato di questo concetto basilare nella visione della donna di Paradiso è tuttavia ancor più sottolineato da Verdi utilizzando un intreccio ritmico che sospende il tempo sulla parola simbolica: fra il disegno dei soprani (una scala cromatica discendente di note di uguale valore, a distanza di quarta eccedente fra le due voci, stridentemente dissonanti e di difficile intonazione) e quello dei contralti (una linea spezzata di salti, ritmicamente disposti in sincope, creando una specie di dissonanza ritmica) si verifica un effetto contraddittorio, la potenza della "benignità" è come spezzata, forte ma tempo stesso indebolita da questa grammatica musicale tanto inusuale e dissonante (es. 7). L'interpretazione offerta, il "comento" di Verdi al testo di Dante non è semplice e merita qualche riflessione: ipotizzo 
che sia da collegare a quella posizione scettica e pessimista che ha minato i suoi ultimi anni, e che abbiamo già presagito nella Ave Maria per soprano e archi. Ciò che è del tutto chiaro, tuttavia, è che Verdi non sta semplicemente "intonando una poesia" come fanno tutti i compositori di romanze e musica vocale da camera italiani in questo periodo (che usano infatti testi poetici meno profondi). Al contrario Verdi medita in musica, commenta, interpreta e offre una personale posizione etica e spirituale attraverso il proprio linguaggio.

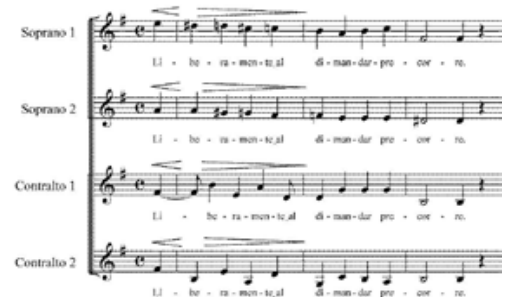

Es. 7, G. Verdi, Laudi alla Vergine Maria ("liberamente”, bb. 66-69)

La conclusione del madrigale spirituale di Verdi riconduce l'attenzione alla simbologia ternaria, già ricordata ed esemplificata in inizio. Anzitutto le quattro invocazioni che il testo di San Bernardo pone in struttura paratattica ("in te misericordia, in te pietade, in te magnificenza, in te s'aduna...”) sono da Verdi separate in una strutturazione di $3+\mathrm{I}$. Le prime tre chiaramente disposte secondo una simmetria che le unisce idealmente: la prima in Re, la seconda in $\mathrm{Mi}$, la terza in Fa diesis; la quarta invocazione è invece separata e la frase corre avanti non verso la tonalità che sarebbe più prevedibile, il Sol maggiore, ma aperta su punti tonali scivolosi da Si a Do, che riportano dopo il disorientamento al centro tonale iniziale e conclusivo. E chiaramente l'intonazione del testo è compiuta, terminata e organicamente concepita su questa chiusura del discorso; l'armonia a tre parti su cui le voci intonano l'ultima sillaba (bontate) è infatti la medesima dell'inizio ("Vergine madre") e nella medesima disposizione (es. 8).

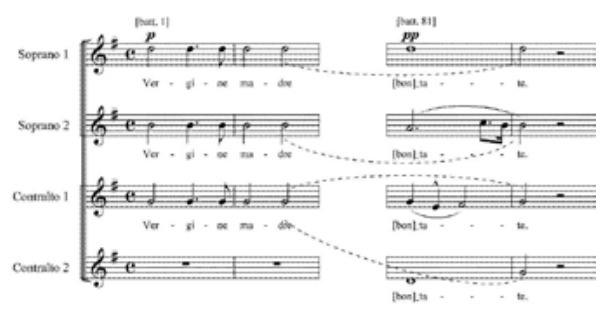

Es. 8, G. Verdi, Laudi alla Vergine Maria (analogia fra l'accordo iniziale e quello su cui termina il testo di Dante [battute I-2 e battute $8 \mathrm{I}-82$ ]) 
In questa conclusione, raggiunto il Sol d'impianto, il compositore aggiunge al testo dantesco due invocazioni ("Ave"), che gli sono necessarie per realizzare un'ultima costruzione sul numero tre. Vediamo come. L'ultima parola della preghiera, sopra esemplificata ("bontate"), è intonata dal primo soprano sulla nota Re; la successiva invocazione "Ave" è intonata tre gradi più in basso, sulla nota Si; ancora tre gradi sotto, sulla nota Sol, è infine intonata l'ultima invocazione. Quindi: tre altezze successive (Re-Si-Sol) sono collegate da una discesa per intervalli di terza, ossia ogni volta tre suoni più in basso. La stessa relazione di terza segna il percorso armonico di questa sezione conclusiva: la prima cadenza ("bontate") è in Sol maggiore, la seguente invocazione "Ave" è in Si maggiore; di nuovo in Sol infine l'ultima ripetizione "Ave": Sol - Si - Sol, come mostra l'esempio seguente (es. 9):

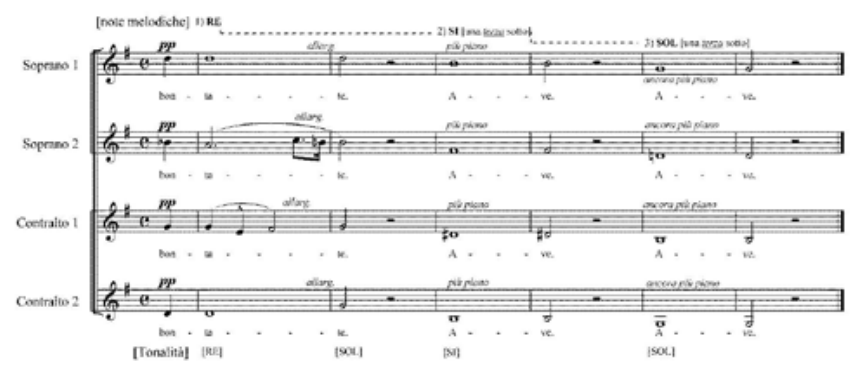

Es. 9, G. Verdi, Laudi alla Vergine Maria (bb. 8I-86)

Nel canto conclusivo della Commedia la preghiera di Bernardo partecipa di quella luminosa smaterializzazione, di quel crescendo di luce che la parola non può ripetere e i sensi non possono sostenere. Verdi, tuttavia, ci offre un commento attraverso la composizione, che non sembra semplicemente ripetere questa esperienza mistica; il compositore, al contrario, con i mezzi del suo linguaggio propone uno sguardo dal punto di vista dell'uomo, del mondo, del dubbio terreno, come nell'Ave Maria e nel Requiem; uno sguardo "dal di qua", senza illusioni e senza certezze fideistiche. Questa conclusione in pianissimo, sebbene apparentemente pacificata, sebbene riporti all'attenzione la simbologia ternaria con efficace chiarezza, non ha nulla di quegli effetti di luminosità crescente, non evoca affatto la mistica perdita di coscienza nella luce divina, nulla del senso di elevazione del canto dantesco. Certo, all'ascolto le Laudi di Verdi suscitano il senso della meditazione intima, dal loro ascolto emerge un umanissimo dubbio, forse anche un disorientamento dell'uomo inerme e solo davanti a una fede che cerca ("dimanda", per usare le parole di Dante), ma che forse non trova. Come Verdi stesso, anche l'io lirico delle Laudi vorrebbe credere, ma non viene investito da quell'onda luminosa che travolge Dante nell'ultimo canto. È una forma di spiritualità desiderosa di una fede di cui 
però non esistono certezze, lasciata in un universo di dubbi col terrore del vuoto e del nulla, ma non scettica né tanto meno dichiaratamente atea; ed è la stessa forma di spiritualità tragica, perché solo terrena, che circola anche nei due Pezzi sacri che circondano le Laudi dantesche, i già ricordati Stabat Mater e il Te Deum.

Le Laudi alla Vergine Maria concludono quindi il rapporto di Verdi con Dante, che era iniziato nel lontano I839 con l'Oberto conte di San Bonifacio e il personaggio protagonistico di Cuniza. Ma le Laudi pongono fine anche alla lunga storia del rapporto fra Dante e i musicisti nell'Ottocento, che era iniziato con Nicola Zingarelli (Il conte Ugolino, cantata per una voce e archi I804) e Gaetano Donizetti (Il conte Ugolino, cantata per basso e pianoforte, I828).

Ed è molto significativo che il tardo Verdi a fine Ottocento sia il primo ad affrontare il testo dantesco con l'intenzione di fornirne un commento attraverso il linguaggio musicale, e non solo di intonarlo in modo estetico, non di sonorizzarne alcune immagini o emozioni, ma per porsi criticamente di fronte a un testo tanto complesso dal punto di vista filosofico e spirituale, cosa del tutto assente dalle intenzioni di tutti gli altri compositori italiani ottocenteschi che abbiano affrontato testi danteschi.

Per meglio spiegare questo fenomeno, questo nuovo atteggiamento critico da parte di Verdi, occorre qui ricordare che negli ultimi decenni dell'Ottocento si assiste ad una generale riconsiderazione di Dante nella cultura italiana: nella Nuova Italia sempre più chiaramente si sviluppa un processo di diffusione della sua figura e della sua poesia con pesanti motivazioni nazionalistiche e populiste. Ma l'ultra-ottantenne Verdi non segue affatto questa corrente e si incammina su una strada del tutto solitaria e contro corrente, verso la meditazione neo-madrigalistica, per cui ricupera la scrittura per voci reali a cappella, secondo un'antica tradizione nazionale del tutto in controtendenza rispetto alla populistica nazionalizzazione del Dante monumentale.

A ben considerare, quindi, questa situazione è piuttosto singolare: il compositore che ancor oggi molti ritengono la massima espressione del popolo italiano più istintivo e meno acculturato, il simbolo del Risorgimento popolare, si indirizza nella sua tarda età in direzione esattamente opposta al populismo già in atto nei governi fra Crispi e Giolitti. E la strumentalizzazione populista, il Dante del popolo come il Verdi per le masse, che arriverà a effetti per entrambi disastrosi nei decenni centrali del Novecento, è un rischio che evidentemente il compositore già presentiva. La riduzione dei mezzi espressivi e l'approfondimento dei mezzi critico-interpretativi nelle Laudi, il loro quasi ascetico impianto ideale, sono la risposta in senso frontalmente contrario a questa strumentalizzazione populista; e forse da questo, dal sorprendente rinnovamento linguistico, dal non uniformarsi al luogo comune che già faceva di Verdi e Dante i "monumenti dello spirito naziona- 
le", deriva alle Laudi la marginalizzazione, il quasi completo oblio, che dura ancora oggi.

Il Verdi ancora oggi divulgato è quello della "trilogia popolare", il "maestro della rivoluzione italiana" e via di questo passo; e sono sintagmi che nascono in questo scorcio di secolo, quando il compositore guarda ormai in direzioni del tutto divergenti. Anche nella ricezione di Dante le Laudi rappresentano una posizione unica e indipendente del tardo Verdi: se da una parte i compositori di romanze impiegano i più semplici testi del Dante stilnovista per dare un tono elevato alle loro composizioni, senza tuttavia rinnovarne né il linguaggio né l'intenzione compositiva (Ciro Pinsuti, Francesco Paolo Tosti, Augusto Rotoli e molti altri che qui non possono trovare spazio, né d'altronde lo meriterebbero), d'altra parte Verdi propone un Dante da meditare in solitudine, in atteggiamento critico, al fine di confrontarsi con i contenuti filosofici dei suoi testi supremi. Per questo alto obiettivo Verdi ricupera i mezzi linguistici della tradizione nazionale più antica: quella del madrigale spirituale. È lo stesso atteggiamento che possiamo ritrovare nel Carducci "umanista" critico dei testi danteschi; un Carducci che, ritrattosi dalla prassi del saggio interpretativo alla De Sanctis, Tommaseo, Torraca (ma prima di loro anche Foscolo e Mazzini), torna all'edizione di tipo più antico, al commento punto per punto dei singoli passi e delle singole allegorie, abbandonando il commento interpretativo generale. Come abbiamo visto, Verdi fa la stessa cosa, prendendo ogni immagine del testo e trovandone un costrutto musicale che generi un nuovo approfondimento riflessivo punto dopo punto, quasi una chiosatura musicale. Verdi non usa mai la forma strofica, la ripetizione di una parte musicale su testo poetico diverso, il che significherebbe una diminuzione di significato del testo e del travaglio critico dell'esegeta-compositore. Ogni parola, ogni immagine, ogni elemento di speculazione è affrontato e commentato con particolari nuovi e specifici del linguaggio musicale, come una nota esplicativa che aggiunge significato al testo dantesco, ma con un'efficacia e immediatezza sensoriale che nessun chiosatore potrebbe raggiungere con la sola parola. È ciò che accadeva nell'antico madrigale spirituale dell'alta tradizione di Palestrina e della scuola romana cinquecentesca.

In questo senso, e solo in questo senso, non in quello della eccessiva espressione urlata a gola spiegata, non nella superficialità delle dichiarazioni di morale spicciola (quelle massime deprimenti che ancora si leggono su alcuni libri sul Risorgimento italiano come "siam tutti fratelli", "morire per la patria" ecc.), non nell'abbondanza di emozionalità, di lacrime inutili e di sangue da palcoscenico, sta il senso della qualità nazionale di Verdi. E in questo senso, ossia in un senso profondamente filosofico, spirituale, riflessivo e critico, si può individuare una lunga linea di cultura italiana nel percorso che abbiamo suggerito: Dante-Palestrina-Verdi. In questo percorso non c'è nulla né del po- 
pulismo né del nazionalismo intesi nel senso comune e aggressivo dei termini. Quello delle Laudi (e in generale dei Quattro pezzi sacri) è un Verdi filosofo che parla della sua esperienza spirituale, un intellettuale che aspira a trovare la fede, ma la cui vicenda esistenziale, il cui senso tragico della vita gli hanno precluso la via a una fede pacificata. E tutto questo travaglio è stato espresso nella musica, solo nella musica, si è "precipitato" in forma musicale, in linguaggio musicale completamente rinnovato. A sua volta tale rinnovamento del proprio "tardo stile" come una forma di autocritica secondo una dinamica più volte indicata nel "tardo stile" dei grandi artisti. ${ }^{17}$ Questa è la forma di spiritualità critica proposta dall'ultimo Verdi, ed è questa forma di spiritualità critica e spesso sofferta che ha contraddistinto una gran parte dell'Italia risorgimentale, eternamente spezzata fra aspirazione di emancipazione liberale-laica e oscurantismi imposti da diverse forme di autorità.

Verdi liberale? Verdi spirituale? Verdi nazionale? O italiano? Verdi scettico? Nessuno di questi sintagmi, da solo, sarebbe soddisfacente, nessuno esaurirebbe la sua eredità intellettuale e nessuno potrebbe dirsi giusto o errato a priori. Il rapporto con Dante, però, è servito a Verdi per chiarire a se stesso un percorso interiore fatto di grandi aspirazioni, grandi esaltazione ideali, ma soprattutto di grandi delusioni, disillusioni, pessimismo. E proprio quello della disillusione è il tono che predomina in questa fase tarda della produzione di Verdi, dove il Dante teologo ha una funzione centrale. Se mai un intellettuale ha compreso questa funzione viva e attuale di Dante, questo è proprio Verdi, lo stesso Verdi "popolare" che molta critica tendenziosa ha voluto assolutizzare.

\section{BIBLIOGRAFIA}

Agamben, G., 20I0, "Disappropriata maniera", in Categorie italiane. Studi di poetica e di letteratura, Roma-Bari, Laterza (ediz. orig. 199I), pp. 82-95.

Broch, H., 1965, "Lo stile dell'età mitica", in Poesia e conoscenza, Milano, Lerici, 1965, pp. 313-333.

Budden, J., 1992, The Operas of Verdi, I, "From Oberto to Rigoletto", Oxford, Clarendon.

Cescotti, O., 1999, Riccardo Zandonai. Catalogo tematico, Lucca, Lim.

Conati, M., 1983, La bottega della musica. Verdi e la Fenice, Milano, Il Saggiatore.

-, 2000, Interviste e incontri con Verdi, Torino, Edt.

D'Angelo, 20Iо, E., Arrigo Boito drammaturgo per musica. Idee, visioni, forma e battaglie, Venezia, Marsilio.

De Angelis, A., I935, La musica a Roma nel secolo XIX, Roma, Bardi.

I7. Il tema del tardo stile ha origini lontane fra Nietzsche e Adorno; qui si pensa però soprattutto alla trattazione più vicina ai nostri giorni, che passa almeno per Broch 2009; infine per Agamben 2010: 82-95. 
Foscolo, U., I825, Discorso sul testo e su le opinioni diverse prevalenti intorno alla storia e alla emendazione critica della commedia di Dante, Londra, Pickering.

Frantellizzi, S. (a cura di), 20II, Il canto dei poeti, Lugano-Milano, Casagrande.

Gamba da Bassano, B., I828, Serie dell'edizioni de' Testi di Lingua..., Venezia, Tipografia d'Alvisopoli.

-, I839, Serie dei testi di lingua e di altre opere importanti nella italiana letteratura scritte dal secolo XIV al XIX [...] Quarta edizione riveduta, emendata e notabilmente accresciuta, Venezia, Co' Tipi del Gondoliere.

La Via, S., 2007, Nascita dell'armonia triadica, in Storia dei concetti musicali, vol. I, Armonia, Tempo, a cura di G. Borio e C. Gentili, Roma, Carocci, pp. 85-I22.

Mila, M., I980, L'arte di Verdi, Torino, Einaudi.

Ortombina, F., 1992, "Sgombra o gentil. Un dono di Verdi all'amico Delfico", in Studi Verdiani, 8, pp. IO4-II7.

Ottomano, V., 2013, "Oberto, conte di S. Bonifacio", in The Cambridge Verdi Encyclopedia, a cura di Roberta Montemorra Marvin, Cambridge, Cambridge University Press, pp. 308-3II.

Rigoli, L., I825, Saggio di Rime di diversi buoni Autori ec., Firenze, Ronchi e Compagni.

Roglieri, M. A., 200o, "Musical settings of the Commedia, arranged by year of composition", in The Dante Encyclopedia, a cura di Richard Lansing, New York, Garland, pp. 279-290.

-, 2012, "Dante and Nineteenth-Century Music", in Dante in the Long Nineteenth Century. Nationality, Identity, and Appropriation, a cura di Aida Audeh e Nick Havely, Oxford, Oxford University Press, pp. 372-390.

Rostagno, A., 20I0-20II, "Otello, le novità del tardo stile di Verdi”, Studi Verdiani, 22, pp. 27-7I.

Said, E., 2009, Sullo stile tardo, 2a ed., Milano, Il Saggiatore.

Zavadini, G., I948, Donizetti: vita, musiche, epistolario, Bergamo, Istituto Italiano d'Arti grafiche. 